\title{
Modulation of low shear stress-induced eNOS multi-site phosphorylation and nitric oxide production via protein kinase and ERK1/2 signaling
}

\author{
XIANGQUAN KONG, XINLIANG QU, BING LI, ZHIMEI WANG, YUELIN CHAO, \\ XIAOMIN JIANG, WEN WU and SHAO-LIANG CHEN
}

Department of Cardiology, Nanjing First Hospital, Nanjing Medical University, Nanjing, Jiangsu 210006, P.R. China

Received November 19, 2015; Accepted November 10, 2016

DOI: $10.3892 / \mathrm{mmr} .2016 .6060$

\begin{abstract}
Physiological shear stress has been demonstrated to serve an atheroprotective function by stimulating endothelial nitric oxide synthase (eNOS) multi-site phosphorylation. Low shear stress (LSS) serves an atheroprone role by increasing endothelial cell apoptosis and inflammation. The present study assessed whether LSS inhibited nitric oxide (NO) production in human umbilical vein endothelial cells by modulating eNOS phosphorylation and potential signaling pathways. A parallel flow chamber imposed with $2 \mathrm{dyn} / \mathrm{cm}^{2}$ shear stress on endothelial cells was used. Western blotting and 4,5-diaminofluorescein diacetate were used to analyze the protein expression levels and NO production. LSS activated eNOS-Ser1177 and eNOS-Thr495, but inhibited eNOS-Ser633. NO production was decreased after a transient increase at 5 min. LSS-stimulated phosphorylation of eNOS-Ser1177 and -Thr495 were suppressed by the Akt inhibitor, perifosine, and extracellular signal regulated kinases1/2 (ERK1/2) inhibitor, PD98059, respectively. Additionally, the phosphorylation of eNOS-Ser633 inhibited by LSS was restored by the protein kinase A activator, 8-Bromo-cAMP. PD98059 completely inhibited the LSS-induced downregulation of NO production. NO downregulation in response to LSS was intensified by perifosine and was partly inhibited by 8-Bromo-cAMP. These results indicated that LSS-induced activation of ERK1/2/eNOS-Thr495 serves a major role in inhibiting endothelial NO synthase, which may explain the proinflammatory and proatherosclerotic properties of LSS.
\end{abstract}

Correspondence to: Professor Shao-Liang Chen, Department of Cardiology, Nanjing First Hospital, Nanjing Medical University, 68 Changle Road, Nanjing, Jiangsu 210006, P.R. China

E-mail: chmengx@126.com

Key words: shear stress, eNOS, protein kinase, ERK1/2, nitric oxide

\section{Introduction}

Shear stress is the frictional force of blood over the surface of the endothelium. Physiological shear stress has been suggested to serve atheroprotective roles by promoting the production of nitric oxide (NO) and inhibiting apoptosis $(1,2)$. Physiological shear stress appears to serve essential roles in the production of NO by mediating the phosphorylation of endothelial nitric oxide synthase (eNOS). Low shear stress (LSS), an atheroprone factor, upregulates the expression of proinflammatory factors, including adhesion molecules, chemoattractant chemokines and cytokines (3), thereby enhancing injury-induced inflammation. Previous studies have demonstrated that LSS $\left(<5 \mathrm{dyn} / \mathrm{cm}^{2}\right)$ in the vasculature, including the inner curvatures of coronary arteries and near bifurcations, promotes atherogenesis by inhibiting eNOS phosphorylation in such regions $(4,5)$. Several potential phosphorylation sites exist on eNOS, among which Ser1177, Ser633 and Thr495 are most extensively investigated (6). Phosphorylation of eNOS at different regulatory sites serves different roles in the regulation of enzyme activation in response to several stimuli. eNOS can be activated by phosphorylation at Ser1177 or Ser633, and inhibited by phosphorylation at Thr495 in the calmodulin binding domain (7).

It has been generally accepted that exposure of endothelial cells to physiological shear stress stimulates the production of NO from eNOS. However, the molecular mechanisms by which shear stress regulates NO remain controversial. Shear stress activates not only protein kinase $\mathrm{B}$ (Akt), but also numerous other target kinases, including protein kinase $\mathrm{A}$ (PKA), protein kinase $\mathrm{C}$ (PKC), serum- and glucocorticoid- inducible kinase, and p70S6 kinase $(8,9)$. Akt, PKA, PKC or AMP-activated kinase, which were reported to phosphorylate eNOS at Ser1177, Ser633 and Thr495, modulate the specific activation of eNOS and NO synthase in endothelial cells subjected to physiological shear stress $(1,10,11)$. By contrast, another previous study demonstrated that shear stress phosphorylates eNOS-Ser1179 in a Phosphatidylinositol-4,5-bisphosphate 3-kinase (PI3K)and PKA-dependent manner, without involving Akt (12).

Although numerous previous reports have focused on the effect of a physiological shear stress of 12 and $15 \mathrm{dyn} / \mathrm{cm}^{2}$ on different eNOS serine/threonine phosphorylation sites and 
signaling pathways, little information is available regarding their phosphorylation in response to LSS $(1,7,10,11)$. Earlier in vitro studies from our laboratory indicated that the possible involvement of LSS-induced human vascular endothelial cell apoptosis is via Akt signaling (13). Due to the differential effects of the phosphorylation at Ser1177, Thr495 and Ser633 on eNOS activation, the present study firstly aimed to determine how these three phosphorylation levels change in response to LSS by protein kinase or other signaling pathways. Secondly, the present study investigated whether LSS-induced changes in eNOS phosphorylation altered endothelial NO release in the presence and absence of various signaling inhibitors or activators. The present study aimed to further clarify the mechanism of LSS-induced endothelial injury. The results may assist when exploring novel markers to protect endothelial function under LSS.

\section{Materials and methods}

Cell culture. Human umbilical vein endothelial cells (HUVECs) were purchased from American Type Culture Collection (Manassas, VA, USA). The cells were maintained in Dulbecco's modified Eagle medium (Gibco; Thermo Fisher Scientific, Inc., Waltham, MA, USA) containing $1 \mathrm{~g} / \mathrm{l}$ glucose and $10 \%$ fetal bovine serum (Gibco; Thermo Fisher Scientific, Inc.) without antibiotics at $37^{\circ} \mathrm{C}$ in a $5 \% \mathrm{CO}_{2}$ incubator. HUVECs at passage 4-9 were used for the following experiments.

LSS studies. The parallel flow chamber was produced by Shanghai Medical Instrument School, as previously described (14). Briefly, by sandwiching a silicon gasket between two stainless steel plates, cells grown to confluence on coverslips were placed the lower plate and subjected to fluid flow powered by a reciprocal pump at 60 times $/ \mathrm{min}$. The value of shear stress was obtained by modulating the proportion of fluid volume passing the flow chamber. The shear stress used in the present experiments was $2 \mathrm{dyn} / \mathrm{cm}^{2}$.

Reagent and supplies. The ERK1/2 inhibitor, PD98059, and cAMP-dependent PKA activator, 8-Bromo-cAMP, were purchased from Sigma-Aldrich (St. Louis, MO, USA). Primary monoclonal rabbit antibodies against phosphorylated p-eNOS -Thr495/Ser1177 (cat. no. 9574/ cat. no. 9571), eNOS (cat. no. 9586), p-ERK1/2 (Thr202/Tyr204) (cat. no. 4377), ERK1/2 (cat. no. 4695), p-Akt-Thr308/ Ser473 (cat. no. 4058/ cat. no. 4056), Akt (cat. no. 4691) and GAPDH (cat. no. 2118), and the Akt inhibitor, perifosine (cat. no. 14240), were obtained from Cell Signaling Technology, Inc. (Beverly, MA, USA). The primary monoclonal mouse antibody against p-eNOS-Ser633 (cat. no. 612664) was purchased from BD Biosciences (Franklin Lakes, NJ, USA). All antibodies were used at a dilution of 1:1,000.

SDS-PAGE and immunoblotting. The cells were lysed on ice in a cocktail of radioimmunoprecipitation assay buffer $[50 \mathrm{mM}$ Tris- $\mathrm{HCl}$ (pH 7.5), 75 mM NaCl, 15 mM EGTA, 1 mM dithiothreitol, 0.1\% Tween-20, $60 \mathrm{mM}$ glycerophosphate, $1 \mathrm{mM} \mathrm{NaF}$, $0.2 \mathrm{mM}$ sodium orthovanadate and $2 \mathrm{mM}$ sodium pyrophosphate; Beyotime Institute of Biotechnology, Shanghai, China], containing proteinase inhibitor (Sigma-Aldrich) and phosphatase inhibitor (Roche Diagnostics, Basel, Switzerland). Homogenates were centrifuged at $12,000 \mathrm{x} g$ for $20 \mathrm{~min}$ at $4^{\circ} \mathrm{C}$. The protein concentrations were quantified using a bicinchoninic acid protein assay kit, according to the manufacturer's protocol (KeyGen Biotech. Co., Ltd., Nanjing, China).

Aliquots of cell lysates were resolved on a $10 \%$ SDS-PAGE gels and transferred onto a polyvinylidene difluoride membrane (Millipore, Bedford, MA, USA). The membranes were blocked with 5\% FBS in TBST for $2 \mathrm{~h}$ prior to incubation with a $1: 1,000$ dilutions of primary antibody overnight at $4{ }^{\circ} \mathrm{C}$ and then with a 1:2,000 dilutions of secondary antibody conjugated with alkaline phosphatase (cat. no. 7074; Cell Signaling Technology, Beverly, MA, USA) for $1 \mathrm{~h}$ at room temperature. The protein bands were detected using the Immobilon Western HRP Substrate Peroxide Solution (cat. no. WBKLS0500; EMD Millipore, Billerica MA, USA). The intensities of immunoreactive bands were analyzed using Image $\mathbf{J}$ software version 1.43 (National Institutes of Health, Bethesda, MD, USA).

Detection of NO. 4,5-diaminofluorescein diacetate (DAF-2DA), a membrane-permeable probe, enters cells and is converted into a product with green fluorescence in the presence of NO. Ice-cold phosphate-buffered saline (PBS) was incubated with DAF-2DA (Life Technologies; Thermo Fisher Scientific, Inc.) at $37^{\circ} \mathrm{C}$ for $30 \mathrm{~min}$ in the dark. Following incubation, the cells were washed twice with ice-cold PBS and their nuclei were labeled with DAPI. Images were captured using a fluorescence microscope (Olympus IX71; Olympus, Tokyo, Japan) and analyzed using Image J software version 1.43. A total of five fields of view were used per sample. DAPI was used for the total cell count in each field of view. Background fluorescence, which was subtracted from the fluorescent intensity values, was determine by imaging unstained cells. The fluorescent intensity values in other groups were normalized to that of the control group, where the value was set to 1.0.

Statistical analysis. Statistical analysis was performed using SPSS 16.0 (SPSS, Inc. Chicago, IL, USA) with one-way analysis of variance followed by the least significant difference test. The data are presented as the mean \pm standard error of the mean of at least three independent experiments $\mathrm{P}<0.05$ was considered to indicate a statistically significant difference.

\section{Results}

LSS alters the phosphorylation of eNOS at Ser1177, Thr495 and Ser633. The present study first set out to investigate the effect of LSS on the phosphorylation of eNOS at various amino acid residues in HUVECs. A parallel plate flow chamber was used to mimic shear stress at $2 \mathrm{dyn} / \mathrm{cm}^{2}$, and the cells were treated for 5, 15, 30 and $60 \mathrm{~min}$. Cells without LSS treatment served as the control. Western blot analyses were performed using antibodies specific to eNOS phosphorylation at Ser1177, Thr495 and Ser633. As shown in Fig. 1, LSS stimulated the phosphorylation of eNOS-Ser1177, which was similar to the effect of physiological shear stress on Ser1177. Phosphorylation of eNOS-Ser1177 was apparent as early as 5 min after LSS onset and reached maximum by $30 \mathrm{~min}$. 


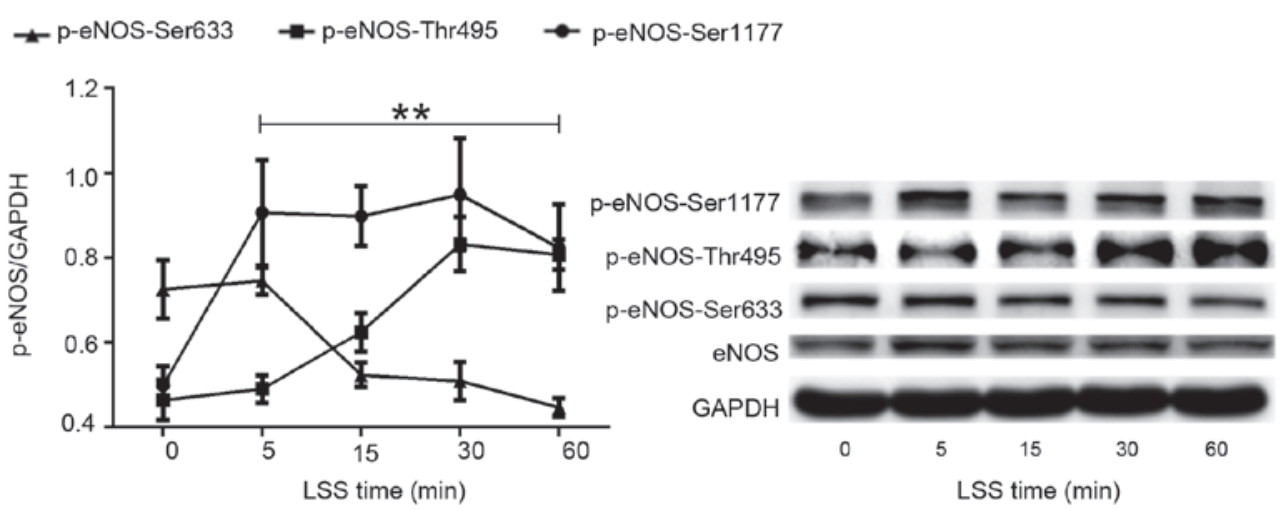

Figure 1. LSS modulates the phosphorylation of eNOS at Ser1177, Thr495 and Ser633. Phosphorylation of eNOS were analyzed by western blotting with antibodies specific for the phosphorylated forms of eNOS at Ser1177, Thr495 and Ser633. The data shown are representative of at least three independent experiments. The phosphorylation of eNOS-Ser1177 and -Thr495 was increased after 5 and 15 min LSS treatment, respectively. The phosphorylation of eNOS-Ser633 was notably reduced after 15 min LSS treatment. The intensity of each band of phosphorylated eNOS was quantified and the levels were normalized to that of the total eNOS. The data are presented as the mean \pm standard error of the mean of $3-4$ independent experiments $\left({ }^{* * *} \mathrm{P}<0.05\right.$, compared with the 0 min time point). p-, phosphorylated-; LSS, low shear stress; eNOS, endothelial nitric oxide synthase.
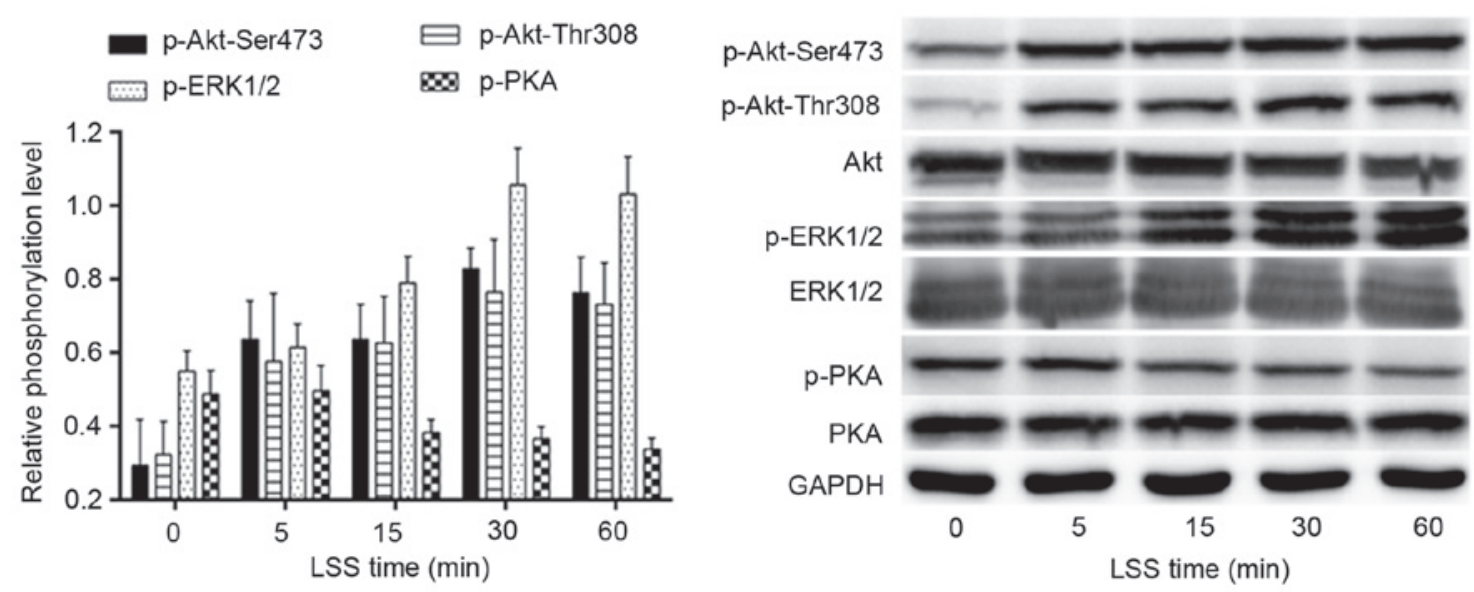

Figure 2. Phosphorylation of Akt, ERK1/2 and PKA in response to LSS. The phosphorylation of Akt-Thr308/Ser473, ERK1/2 and PKA were analyzed by western blotting with antibodies specific for the total and phosphorylated forms of the proteins. The expression levels were normalized to that of the total levels. The data shown are representative of at least three independent experiments. The phosphorylation of Akt-Thr308 and Akt-Ser473 were increased from 5 min LSS exposure, and the phosphorylation of ERK1/2 was elevated from 15 min LSS exposure. By contrast, the phosphorylation of PKA was reduced after $5 \mathrm{~min}$ LSS exposure. The intensity of each band was quantified and the expression levels of the proteins were normalized to that of the total levels. The data are presented as the mean \pm standard error of the mean of 3-4 independent experiments. p-, phosphorylated-; LSS, low shear stress; ERK, extracellular signal-regulated kinase; PKA, protein kinase A; Akt, protein kinase B.

When HUVECs were exposed to LSS, a significant increase in the phosphorylation at Thr495 was observed in a time-dependent manner. LSS-stimulated phosphorylation of Thr495 was later than that of Ser1177. Any significant increase of phosphorylation of eNOS-Thr495 was observed at $15 \mathrm{~min}$ LSS exposure and remained elevated for the $60 \mathrm{~min}$ (Fig. 1).

However, the effect of LSS on eNOS-Ser633 was completely different compared with that of eNOS-Ser1177 and -Thr495. Fig. 1 suggested that 15 min LSS exposure was required to observe a significant decrease of the phosphorylation of eNOS-Ser633. This decrease in expression remained for at least $60 \mathrm{~min}$.

LSS regulates the phosphorylation of Akt-Thr308/Ser473, $E R K 1 / 2$ and PKA. The effects of LSS on the phosphorylation of Akt, ERK1/2 and PKA were determined by western blotting using antibodies specific for each phosphorylated site at various time points. As shown in Fig. 2, the LSS-dependent phosphorylation of Akt-Thr308, as well as Akt-Ser473, began to increase at $5 \mathrm{~min}$ LSS and the phosphorylation of ERK1/2 occurred later than $5 \mathrm{~min}$ LSS. The phosphorylation of Akt-Ser473/Thr308 and ERK1/2 peaked at 30 min; however, PKA demonstrated an adverse tendency with Akt and ERK1/2 in the same time course. The phosphorylation of PKA was reduced after $5 \mathrm{~min}$ LSS. The lowest value may actually be observed outside the 60 min time course.

LSS induces the phosphorylation of eNOS-Ser1177 and eNOS-Ser633 in a protein kinase-dependent manner. The present study next aimed to determine whether phosphorylation of eNOS at Ser1177 and dephosphorylation at Ser633 by LSS are regulated in a protein kinase-dependent manner. Perifosine, an inhibitor of Akt-Ser473 and -Thr308, was used to treat the cells prior to the initiation of LSS for $30 \mathrm{~min}$. Western blot analysis revealed that treatment of HUVECs with perifosine was effective in depressing LSS-dependent 

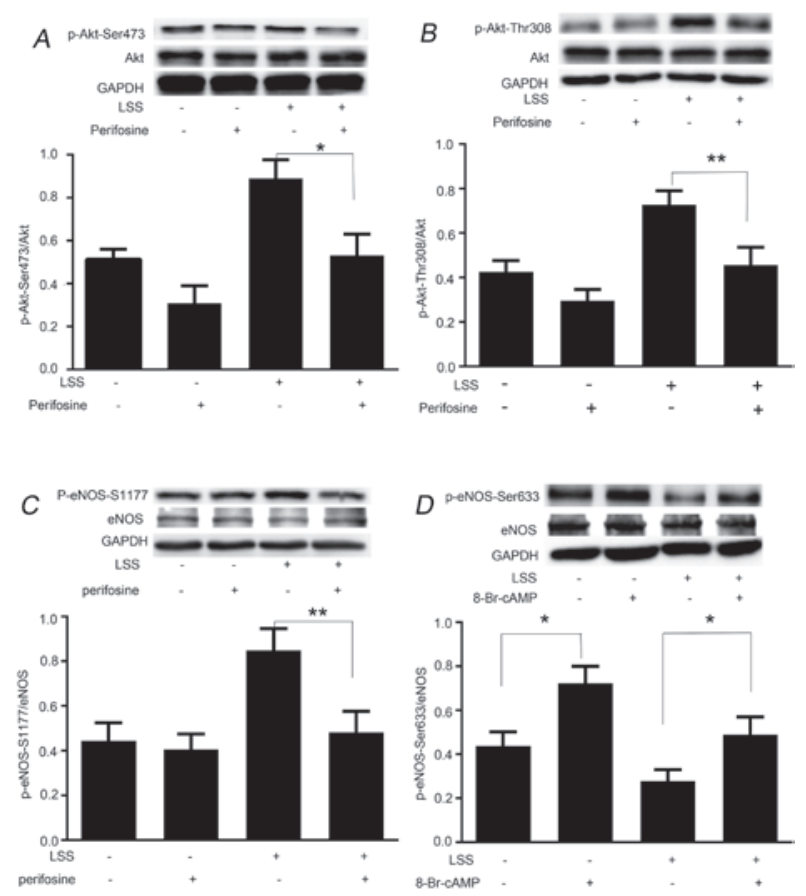

Figure 3. Phosphorylation of eNOS-Ser1177 and -Ser633 in response to LSS via the protein kinase pathway. HUVECs were pretreated with vehicle (DMSO) or $40 \mu \mathrm{mol} / 1 \mathrm{Akt}$ inhibitor (perifosine) for $30 \mathrm{~min}$ prior to exposure to LSS for $30 \mathrm{~min}$. Perifosine significantly inhibited not only the phosphorylation of (A) Akt-Ser473 and (B) Akt-Thr308, but also the phosphorylation of (C) eNOS-Ser1177 following exposure to LSS. (D) 8-Br-cAMP (1 mmol/l) significantly elevated the expression of phosphorylation of eNOS-Ser633, which was decreased by LSS treatment in HUVECs. The intensity of each band was quantified and the protein expression levels were normalized to that of the total levels. The data are presented as the mean \pm standard error of the mean of 3-4 independent experiments $\left({ }^{*} \mathrm{P}<0.01\right.$ and $\left.{ }^{* *} \mathrm{P}<0.05\right)$. HUVECs, human umbilical vein endothelial cells; p-, phosphorylated-; LSS, low shear stress; eNOS, endothelial nitric oxide synthase.
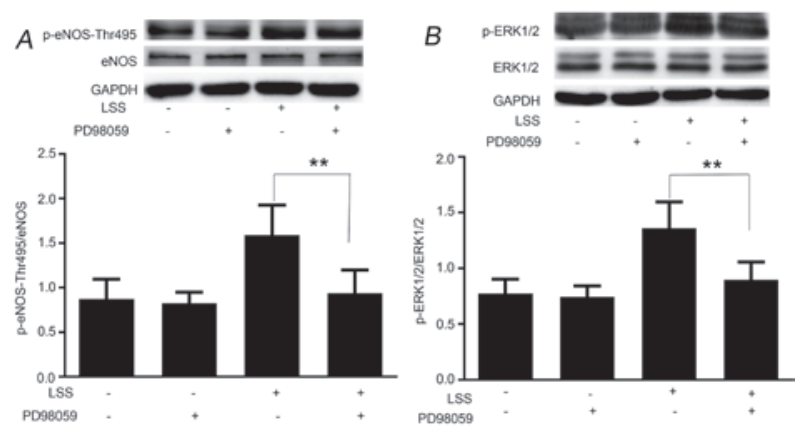

Figure 4. LSS induces the phosphorylation of eNOS-Thr495 via the ERK1/2 pathway. HUVECs were pretreated with vehicle (DMSO) or $30 \mu \mathrm{mol} \mathrm{l}^{-1}$ ERK1/2 inhibitor, PD98059, for $30 \mathrm{~min}$ prior to exposure to LSS for $30 \mathrm{~min}$. The phosphorylation of (A) eNOS-Thr495 and (B) ERK1/2 were notably downregulated by PD98059. The intensity of each band was quantified and the expression levels of the proteins were normalized to that of GAPDH. The data are presented as the mean \pm standard error of the mean of 3-4 independent experiments $\left({ }^{* *} \mathrm{P}<0.05\right)$. HUVECs, human umbilical vein endothelia cells; p-, phosphorylated-; LSS, low shear stress; eNOS, endothelial nitric oxide synthase; ERK, extracellular signal-regulated kinase.

phosphorylation of not only Akt-Ser473 (Fig. 3A), but also Akt-Thr308 (Fig. 3B). Perifosine also significantly inhibited the LSS-dependent phosphorylation of eNOS-Ser1177 (Fig. 3C); however, did not suppress LSS-induced attenuation of phosphorylation of eNOS-Ser633 (data not shown). As shown in Fig. 3D, treatment of the cells with 8-Br-cAMP for 30 min significantly recovered the phosphorylation level of eNOS-Ser633. In addition, 8-Br-cAMP significantly elevated static phosphorylation of eNOS-Ser633 and -Ser1179; however, not eNOS-Thr495 (data not shown).

LSS phosphorylates eNOS-Thr495 via the ERK1/2 pathway. To examine whether the LSS-evoked ERK1/2 is upstream of eNOS-Thr495, HUVECs were pretreated with the ERK1/2 inhibitor, PD98059, for $30 \mathrm{~min}$. As shown in Fig. 4, PD98059 was able to reduce the phosphorylation of eNOS-Thr495 (Fig. 4A) by inhibiting the phosphorylation of ERK1/2 (Fig. 4B); however, did not reduce the phosphorylation of eNOS-Ser1177 in response to LSS (data not shown). The basic phosphorylation levels of eNOS-Ser1177 and eNOS-Thr495 remained unchanged by PD98059 (data not shown).

ERK1/2/eNOS-Thr495 is a critical signaling pathway for LSS-mediated NO synthase decrease. NO production under LSS was measured using the NO-specific fluorescent dye DAF-2 DA. The results demonstrated that LSS rapidly stimulated NO release in a transient manner with a maximum at $5 \mathrm{~min}$; however, after $5 \mathrm{~min}$, NO production was observed a time-dependent attenuation (Fig. 5A and B). A total of $30 \mathrm{~min}$ pretreatment with PD98059 completely inhibited LSS-induced suppression of NO production (Fig. 5C). In order to clarify the signaling mechanisms of eNOS-Ser1177 and eNOS-Ser633 on NO production mediated by LSS, the HUVECs were pretreated with perifosine or 8 -Br-cAMP for 30 min prior to a 30 min exposure to LSS. The results demonstrated that 8-Br-cAMP significantly abolished negative effect of LSS on NO production to a certain degree, although NO production was not restored to the control levels (Fig. 5D). Perifosine modestly aggravated LSS-induced NO drop (Fig. 5E).

\section{Discussion}

Our previous findings demonstrated that LSS induces human vascular endothelial cell apoptosis via Akt signaling (13). The present study investigated the phosphorylation of eNOS at Ser1177, Thr495 and Ser633, and the possible mechanisms in HUVECs in response to LSS. The results revealed that activation of Akt/eNOS-Ser1177 can completely reverse LSS-induced NO synthase decrease via the PKA/eNOS-Ser633 pathway. Downregulation of endothelial NO predominantly depended on phosphorylation of eNOS-Thr495 via an ERK1/2 mechanism, since PD98059 completely inhibited the NO downregulation induced by LSS.

It is well known that phosphorylation of eNOS-Ser1177 stimulates eNOS activation in response to various physiological stimuli, including bradykinin, shear stress and vascular endothelial growth factor, which activate eNOS in a $\mathrm{Ca}^{2+}$-dependent or $\mathrm{Ca}^{2+}$-independent manner $(10,11)$. It has been previously demonstrated that physiological shear stress, as an atheroprotective factor, increases the phosphorylation of eNOS-Ser1177 and NO production (12). The present data suggested LSS, as a atheroprone factor, also stimulated the phosphorylation of eNOS-Ser1177, while NO level decreased after a transient increase at $5 \mathrm{~min}$ LSS. The present study 
A

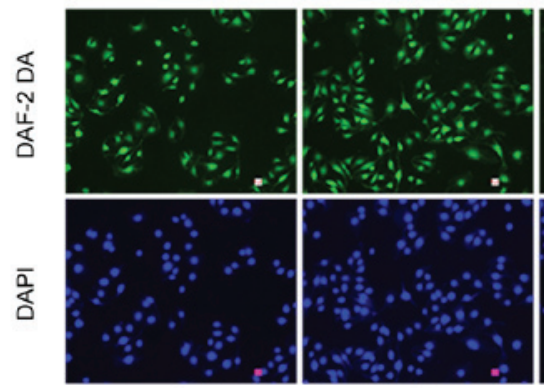

0

B

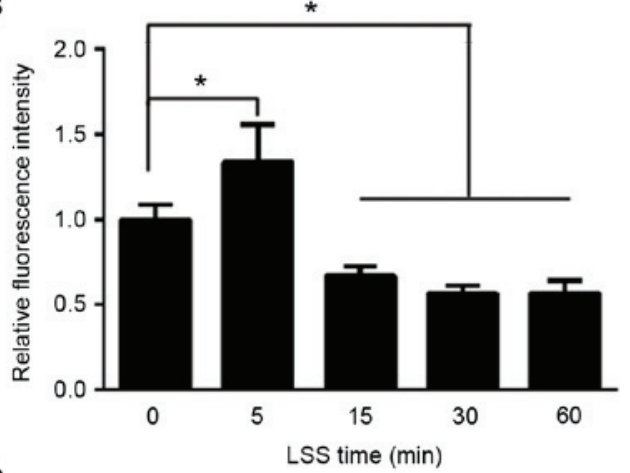

D

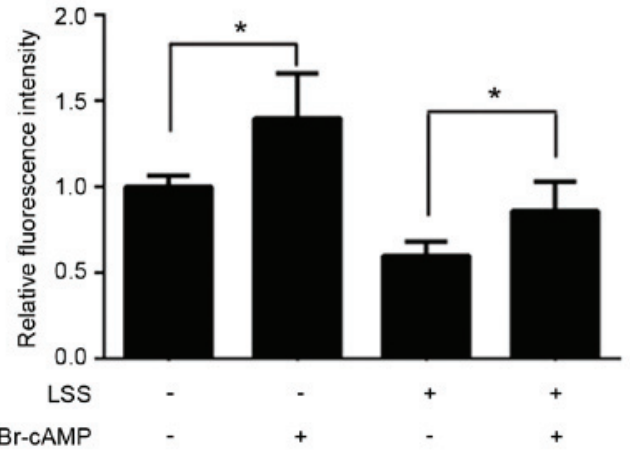

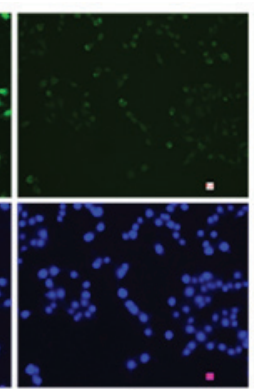

15

LSS time ( $\mathrm{min})$

C

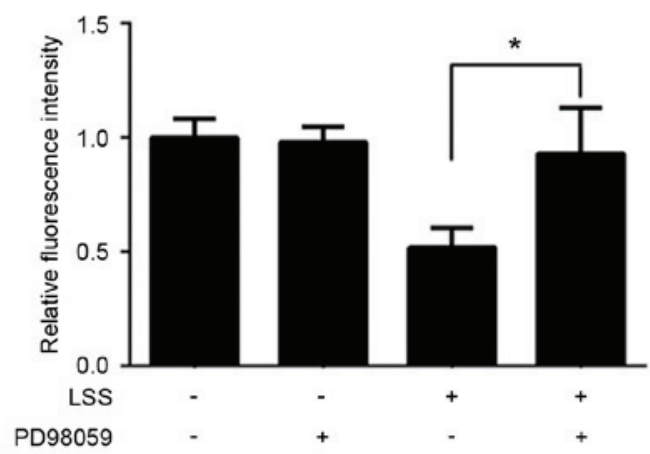

E

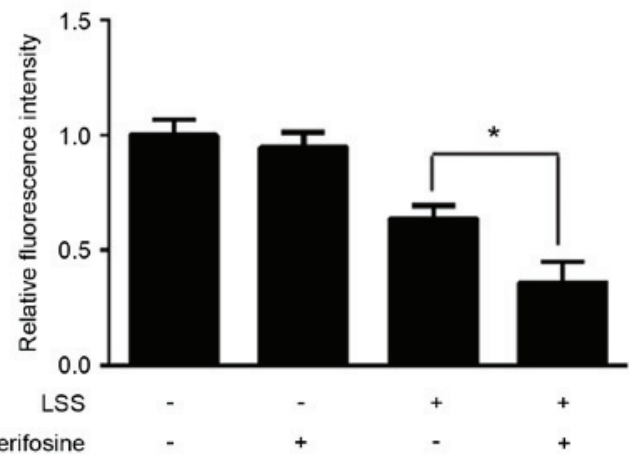

Figure 5. Effect of LSS on NO production and signaling mechanisms in HUVECs. (A and B) HUVECs were exposed to LSS for 0, 5, 15, 30 and 60 min. NO were stained using the NO-specific fluorescent dye DAF-2 DA (scale bar, $25 \mu \mathrm{m}$ ). Although LSS caused a transient NO output growth at 5 min, NO production demonstrated a notable time-depended decrease from $15 \mathrm{~min}$ in response to LSS. The HUVECs were pretreated with vehicle (DMSO) or $30 \mu \mathrm{mol} / 1$ ERK1/2 inhibitor, PD98059, and the NO production was assessed. (C) NO output, which was inhibited by LSS treatment, was completely recovered to the control levels following treatment with PD98059. (D) The PKA activator, 8-Br-cAMP (1 mmol/l), not only partially inhibited LSS-induced NO production decline, but also stimulated static HUVECs NO production, which was not observed following PD98059 and perifosine treatment. (E) The reduction in NO production was intensified following treatment with $40 \mu \mathrm{mol} / \mathrm{l} \mathrm{Akt} \mathrm{inhibitor,} \mathrm{perifosine.} \mathrm{The} \mathrm{data} \mathrm{are} \mathrm{presented} \mathrm{as} \mathrm{the} \mathrm{mean} \pm$ standard error of the mean of 3-4 independent experiments ("P<0.01). HUVECs, human umbilical vein endothelial cells; LSS, low shear stress; NO, nitric oxide; PKA, protein kinase A; Akt, protein kinase $\mathrm{B}$.

accounted the alternation of NO level for the differential change of eNOS multi-site phosphorylation. Transient increase of $\mathrm{NO}$ is likely to be a protective effect of endothelial cells at the beginning of harmful stimuli.

Physiological shear stress was reported to phosphorylate eNOS-Ser1179 by a PI3K mechanism in bovine aortic endothelial cells (15). However, other experiments have demonstrated that eNOS-Ser1179 was not directly phosphorylated by PI3K. It was concluded that PI3K caused activation of phosphoinositide-dependent kinase-1 (PDK1), which in turn stimulated downstream PKA, which directly or indirectly phosphorylated eNOS-Ser1179 in bovine aortic endothelial cells (12). Whether the PI3K/PDK1 pathway regulates PKA and other protein kinases, in response to LSS, remains to be determined. In the present study, LSS stimulated the phosphorylation of PI3K within 5 min. Inhibiting PI3K with wortmannin or LY294002 suggested that PI3K was not involved in regulating eNOS phosphorylation at Ser1177, Ser633 or Thr495 (data not shown).

Another significant finding of the present study was that LSS promoted the dephosphorylation of eNOS-Ser633 and PKA in a time-dependent manner. The PKA activator, 8-br-cAMP, restored the level of phosphorylation of eNOS-Ser633 inhibited by LSS. This result illustrated that eNOS-Ser633 as a protective amino acid residue is dephosphorylated via the dephosphorylation of PKA by LSS. Numerous experiments under physiological stimuli, including shear stress, have suggested that PKA increased the level of phosphorylation at the site of eNOS-Ser633 or other sites, and NO production (16-19). These results suggested that the effects 
of shear stress on the phosphorylation of eNOS-Ser633 and PKA depend on shear stress value. In other words, phosphorylation of eNOS-Ser633 begins to reduce with the decline of the value of shear stress.

The present data provided certain insights how LSS has an effect on the phosphorylation of eNOS-Thr495. The ERK1/2 inhibitor, PD98059, completely prevented the phosphorylation of eNOS-Thr495 caused by LSS. It demonstrated LSS-stimulated phosphorylation of eNOS-Thr495 is via ERK1/2 pathway. However phosphorylation of eNOS-Ser1177 promoted NO output and dephosphorylation of eNOS-Ser633 downregulated NO output under LSS. In addition, NO output recovered to almost control degree with PD98059 inhibiting the phosphorylation of eNOS-Thr495. These findings suggested that LSS-induced function of eNOS-Ser1177 and -Ser633 is cancelled out by each other. NO synthase decrease following LSS predominantly depends on the phosphorylation of eNOS-Thr495 modulated by ERK1/2. The effect of physiological shear stress on the phosphorylation of eNOS-Thr495 indicated certain contradictory results in certain previous studies $(16,20)$. Barauna et al (20) revealed that inhibition of shear stress-induced ERK activation leads to increasing eNOS activation by eNOS-Thr495 dephosphorylation in human saphenous vein endothelial cells. However, other findings suggest no changes on the phosphorylation status of eNOS-Thr497 by shear stress in bovine aortic endothelial cells (16). It is possible that the different species of cells are one of underlying reasons for such differences, and also different stimuli appear to elicit distinct changes of phosphorylation of eNOS-Thr495 and -Ser1177. Although, phosphorylation of eNOS-Thr495 and -Ser1177 were increased by LSS in the present study, NO release was abolished in a time-dependent manner after 5 min LSS. A simple interpretation of this result is that phosphorylation of eNOS-Thr495 is a predominant factor that promotes endothelial injury induced by LSS.

Certain previous experiments have demonstrated that phosphorylation of eNOS at Thr495 is PKC-dependent (21-24). In order to elucidate a potential upstream signaling molecule of ERK1/2, PKC, another member of the protein kinase familiar, was investigated with phospho-specific anti-PKC antibodies (data not shown); however no stimulus-related changes in the association of classical PKC and eNOS-Thr495 were observed. One reason is that PKC has nothing to do with the phosphorylation of eNOS-Thr495 promoted by LSS. The other reason is that other subtypes of PKC that are excluded in the present study are involved in phosphorylating eNOS-Thr495.

In conclusion, the present study found that LSS changes the phosphorylation of eNOS at Ser1177, Thr495 and Ser633. Phosphorylation of eNOS-Ser1177 and dephosphorylation of eNOS-Ser63 under LSS are regulated in a protein kinase-dependent manner. The activation of Akt is responsible for the phosphorylation of eNOS-Ser1177 and elevation of NO in 5 min. LSS-stimulated NO release via Akt/eNOS-Ser1177 is neutralized by dephosphorylation of eNOS-Ser633, derived from deactivated PKA. Changes in the phosphorylation at eNOS-Thr495 resulted in a decrease in NO and endothelial injury. This may explain why LSS is an atheroprone factor. The present results provided important insights and suggested that ERK1/2 inhibition can restore endothelial NO production under LSS.

\section{Acknowledgements}

The present study was supported by the National Natural Science Foundation of China (no. 81270191). The authors would like to thank Professor Yu-Lin Gu (Nature-Think Company, Shanghai, China) for technical support of the mechanics of the parallel flow chamber.

\section{References}

1. Tarbell JM, Shi ZD, Dunn J and Jo H: Fluid mechanics, arterial disease, and gene expression. Annu Rev Fluid Mech 46: 591-614, 2014.

2. Resnick N, Yahav H, Shay-Salit A, Shushy M, Schubert S, Zilberman LC and Wofovitz E: Fluid shear stress and the vascular endothelium: For better and for worse. Prog Biophys Mol Biol 81: 177-199, 2003.

3. Chatzizisis YS, Coskun AU, Jonas M, Edelman ER, Feldman CL and Stone PH: Role of endothelial shear stress in the natural history of coronary atherosclerosis and vascular remodeling: Molecular, cellular, and vascular behavior. J Am Coll Cardiol 49: 2379-2393, 2007.

4. Gimbrone MA Jr, Topper JN, Nagel T, Anderson KR and Garcia-Cardeña G: Endothelial dysfunction, hemodynamic forces, and atherogenesis. Ann N Y Acad Sci 902: 230-239, 239-240, 2000.

5. Cunningham KS and Gotlieb AI: The role of shear stress in the pathogenesis of atherosclerosis. Lab Invest 85: 9-23, 2005.

6. Ramadoss J, Pastore MB and Magness RR: Endothelial caveolar subcellular domain regulation of endothelial nitric oxide synthase. Clin Exp Pharmacol Physiol 40: 753-764, 2013.

7. Kolluru GK, Siamwala JH and Chatterjee S: eNOS phosphorylation in health and disease. Biochimie 92: 1186-1198, 2010.

8. Toker A and Newton AC: Cellular signaling: Pivoting around PDK-1. Cell 103: 185-188, 2000.

9. MaroskiJ, VorderwülbeckeBJ,FiedorowiczK,DaSilva-AzevedoL, Siegel G, Marki A, Pries AR and Zakrzewicz A: Shear stress increases endothelial hyaluronan synthase2 and hyaluronan synthesis especially in regard to an atheroprotective flow profile. Exp Physiol 96: 977-986, 2011.

10. Michell BJ, Harris MB, Chen ZP, Ju H, Venema VJ, Blackstone MA, Huang W, Venema RC and Kemp BE: Identification of regulatory sites of phosphorylation of the bovine endothelial nitric-oxide synthase at serine 617 and serine 635. J Biol Chem 277: 42344-42351, 2002.

11. Dimmeler S, Fleming I, Fisslthaler B, Hermann C, Busse R and Zeiher AM: Activation of nitric oxide synthase in endothelial cells by Akt-dependent phosphorylation. Nature 399: 601-605, 1999.

12. Boo YC, Sorescu G, Boyd N, Shiojima I, Walsh K, Du J and Jo H: Shear stress stimulates phosphorylation of endothelial nitric-oxide synthase at Ser1179 by Akt-independent mechanisms: Role of protein kinase A. J Biol Chem 277: 3388-3396, 2002.

13. Zhang J, Wang Z, Zuo G, Li B, Zhang J, Tian N and Chen S: Low shear stress induces human vascular endothelial cell apoptosis by activating Akt signal and increasing reactive oxygen species. Nan Fang Yi Ke Da Xue Xue Bao 33: 313-317, 2013.

14. Wang Z, Zhang J, Li B, Gao X, Liu Y, Mao W and Chen SL: Resveratrol ameliorates low shear stressinduced oxidative stress by suppressing ERK/eNOSThr495 in endothelial cells. Mol Med Rep 10: 1964-1972, 2014.

15. Devika NT and Jaffar Ali BM: Analysing calcium dependent and independent regulation of eNOS in endothelium triggered by extracellular signalling events. Mol Biosyst 9: 2653-2664, 2013.

16. Boo YC, Hwang J, Sykes M, Michell BJ, Kemp BE, Lum H and Jo H: Shear stress stimulates phosphorylation of eNOS at Ser(635) by a protein kinase A-dependent mechanism. Am J Physiol Heart Circ Physiol 283: H1819-H1828, 2002.

17. Walther S, Pluteanu F, Renz S, Nikonova Y, Maxwell JT, Yang LZ, Schmidt K, Edwards JN, Wakula P, Groschner K, et al: Urocortin 2 stimulates nitric oxide production in ventricular myocytes via Akt- and PKA-mediated phosphorylation of eNOS at serine 1177. Am J Physiol Heart Circ Physiol 307: H689-H700, 2014.

18. Hu Z, Xiong Y, Han X, Geng C, Jiang B, Huo Y and Luo J: Acute mechanical stretch promotes eNOS activation in venous endothelial cells mainly via PKA and Akt pathways. PLoS One 8: e71359, 2013 
19. Yang C, Talukder MA, Varadharaj S, Velayutham M and Zweier JL: Early ischaemic preconditioning requires Akt- and PKA-mediated activation of eNOS via serine1176 phosphorylation. Cardiovasc Res 97: 33-43, 2013.

20. Barauna VG, Mantuan PR, Magalhaes FC, Campos LC and Krieger JE: AT1 receptor blocker potentiates shear-stress induced nitric oxide production via modulation of eNOS phosphorylation of residues $\operatorname{Thr}(495)$ and Ser(1177.). Biochem Biophys Res Commun 441: 713-719, 2013.

21. Chen F, Kumar S, Yu Y, Aggarwal S, Gross C, Wang Y, Chakraborty $\mathrm{T}$, Verin AD, Catravas JD, Lucas R, et al PKC-dependent phosphorylation of eNOS at T495 regulates eNOS coupling and endothelial barrier function in response to $\mathrm{G}+$ -toxins. PLoS One 9: e99823, 2014.
22. Jiang X, Yang F, Tan H, Liao D, Bryan RM Jr, Randhawa JK, Rumbaut RE, Durante W, Schafer AI, Yang X and Wang H: Hyperhomocystinemia impairs endothelial function and eNOS activity via PKC activation. Arterioscler Thromb Vasc Biol 25: 2515-2521, 2015

23. Sakata K, Kondo T, Mizuno N, Shoji M, Yasui H, Yamamori T, Inanami $\mathrm{O}$, Yokoo $\mathrm{H}$, Yoshimura $\mathrm{N}$ and Hattori Y: Roles of ROS and PKC- $\beta$ II in ionizing radiation-induced eNOS activation in human vascular endothelial cells. Vascul Pharmacol 70: 55-65, 2015.

24. Chu S and Bohlen HG: High concentration of glucose inhibits glomerular endothelial eNOS through a PKC mechanism. Am J Physiol Renal Physiol 287: F384-F392, 2004. 